\title{
Oral Status of a Pediatric Patient with Merosine Negative Congenital Muscular Dystrophy
}

\author{
Livia Roberta Piedade De Oliveira ${ }^{1}$, Ivete Pomarico Ribeiro De Souza ${ }^{1}$ and Carla Martins ${ }^{2}$ \\ ${ }^{1}$ Department of Pediatric Dentistry and Orthodontics, Universidade Federal do Rio de Janeiro, Brazil \\ ${ }^{2}$ Department of Dental Clinic, School of Dentistry, Universidade Federal do Rio de Janeiro, Brazil
}

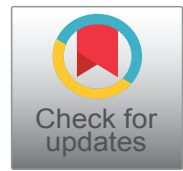

*Corresponding author: Livia Roberta Piedade De Oliveira, Department of Pediatric Dentistry and Orthodontics, School of Dentistry, Universidade Federal do Rio de Janeiro, Rio de Janeiro, RJ, Brazil, E-mail: liviaroberta@yahoo.com.br

\begin{abstract}
Merosine Deficient Congenital Muscular Dystrophy (MDCMN) is a rare subtype of Muscular Dystrophy and it is defined as generalized hypotonic muscular syndrome. This case report aimed to describe the oral status of a six-yearold girl with MDCMN. The family's and child's chief complaint was bad breath. The child, who presented normal intelligence, had physical disability with mobility impairment and so, used wheelchair. Her feeding was done mostly through gastric feed tube, however, the child used to eat soft food, liquids and some candies by mouth. The extraoral examination showed facial hypotonic muscles, inadequate labial sealing and bimaxillary discrepancies. While the intraoral exam revealed mixed dentition with infraocclusion of both second primary molars and class III molar occlusion. Although the child was caries-free, her oral hygiene was deficient and dental calculus were observed on the lower teeth. In addition, the intrusion of the right upper primary canine. Radiographic findings included agenesis of the lower second premolar bud. Mechanical debridation, prophylaxis and fluoride application were performed. Besides, both mother and child were instructed and motivated in relation to oral hygiene (tongue scrapping) and diet, in order to minimize halitosis. Muscular Dystrophy brings to the patients several complications, including oral manifestations. Thus, oral health plays an important role and the dentist needs to pay attention, in order to contribute the improvement of the quality of life of these patients.
\end{abstract}

\section{Keywords}

Congenital muscular dystrophy, Merosine negative, Oral status, Child

\section{Introduction}

Muscular dystrophy (MD) represents a group of dis- eases and its etiology varies depending on the type of dystrophy, which it may differ through different molecular mechanisms, as do the incidence and impact on a patient's quality of life. The most common type, Duchenne MD, occurs with an incidence of approximately 1 in 3,500 live-born male infants [1]. However, there are rarer forms, such as Congenital MD (CMD), which has an autosomal recessive inheritance pattern. In this case, the incidence is only 0.7 per 100,000 live births [2]. Each type of CMD is sub classified based on the specific molecular defect or deficiency. Merosin deficient CMD (MDCMD) is a severe form of MD that is manifested by an inability to ambulate independently secondary to muscle weakness/contractures and results from a deficiency of laminin-2 chains (merosin) because of mutations in the LAMA2 gene located on chromosome $6 q 22$, which accounts for up to $30 \%$ of CMD cases in European countries [3].

CMD is characterized by severe muscle hypotonia (first noticeable at birth or within the first months of life), generalized muscle weakness, and muscle contractures of varying severity that result in delayed or missed developmental motor milestones [1]. Patients with muscular dystrophy present similar oral characteristics. One of them is apertognathia, which is the term used to describe the bimaxillary discrepancies characterized by wide anterior open bite absence of the sucking reflex, macroglossia and limited maximal incisal opening are some of the characteristics presented by patients with muscular dystrophy [1,4-7]. In our report, we aimed to describe the oral status of a 6-year-old child with MDCMD.

Citation: De Oliveira LRP, De Souza IPR, Martins C (2018) Oral Status of a Pediatric Patient with Merosine Negative Congenital Muscular Dystrophy. Int J Pediatr Res 4:034. doi.org/10.23937/24695769/1510034

Accepted: June 20, 2018: Published: June 22, 2018

Copyright: (C) 2018 De Oliveira LRP, et al. This is an open-access article distributed under the terms of the Creative Commons Attribution License, which permits unrestricted use, distribution, and reproduction in any medium, provided the original author and source are credited. 


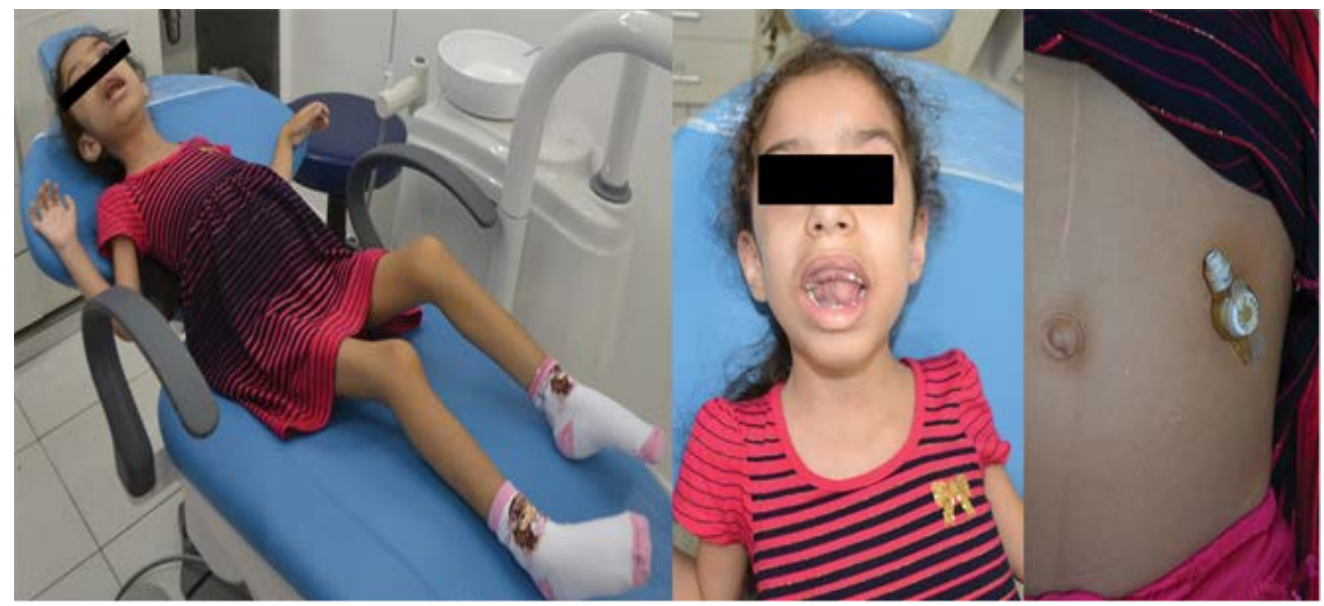

Figure 1: a) Child on the dental chair; b) Extra-oral aspect; (c) Gastric Fed Tube.



Figure 2: Aspect of maximum intercuspation occlusion and wide anterior open bite observed.

\section{Case Report}

A 6-year-old Caucasian girl diagnosed with Congenital Muscular Dystrophy Merosine - Negative was referred to the Clinic from the Department of Pediatric Dentistry at Federal University of Rio de Janeiro, after her parents complained to her endocrinologist from the Pediatric Hospital at the same institution she had bad breath. The girl is twin child with of a non-consanguineous family and her twin sister presented no systemic disease.

During the anamnesis, the parents revealed that the child's medical history was significant for intercurrences during birth, when she presented with low weight and was kept in the incubator for babies about 55 days. The child presented high glucose serum levels, and was diagnosed at birth as monogenic diabetes, a rare subtype of diabetes mellitus, which undertake babies under 6-months-old and it is transitory. From birth to age of 4-months, the child had sucking reflex and used pacifier. Natural feeding was normally performed but at 10-months-old, the child stopped swallowing and sucking, so she was unable anymore to be fed by her mother. Thus, she underwent surgery of gastric feeding tube placement. Due recurrent infections (pneumonia and sinusitis), the child was frequently submitted to antibioticoteraphy. At the age of 1-year-old she presented myopathy and at 2-years-old, she was examined through imagining magnetic resonance, in order to look for some neurological disturbances, since her head control was not perfect, nor could she stand or walk at that time. At 3-years-old, a biopsy was performed, and Dystrophy was diagnosed, however, only at 6-years-old she was submitted to genetic exam and merosin-deficient CMD was diagnosed.

Her condition was associated with normal intelligence and the child attends special school and expresses herself very well, liked to watch TV and to play with her sister. Regarding to eating habits, most of it is inserted through the gastric tube, due to the dystrophy, which does not allow her to swallow very well. However, the child consumed little soft food, liquid and even candies. She was still followed by Endocrinology Department due to diabetes, which was transitory, and she no longer takes any medicine. 
The extraoral clinical examination showed hypotonic facial muscles and inadequate lip coverage, making her a chronic mouth - breathing child (Figure 1). The intraoral examination revealed a wide anterior open bite (15 $\mathrm{mm}$ ) and occlusion only on the first permanent molars, which are in relation Class III, thus leading to anterior interposition of the tongue (Figure 2). The aspect of upper arch is shown in Figure 3a. Infraocclusion of the first primary lower molars were also observed (Figure 3b). Although the child was caries free, she presented dental calculus on every anterior lower lingual and interproximal surfaces.

Furthermore, the child's mother related the girl sustained a traumatic dental injury while she fell into the floor from the height of her wheelchair three months earlier the consultation, causing intrusion of her upper right primary canine (Figure 2). However, the parents had not perceived the tooth intrusion once the child had not claim of pain in this region. Panoramic radiography examination showed apertognathia, absence of a dental element bud (\#45) and infraocclusion of both mandibular first primary molars (Figure 4).

Thus, mechanical debridation, dental prophylaxis and fluoride application were performed. In addition, the importance of rational sugar consumption and improving the frequency of oral hygiene with fluoride dentifrice were highlighted to both child and mother, who were instructed to how to brush her teeth, scrap the tongue and how to use dental floss. Even feeding being usually inserted by the gastric tube, oral hygiene is indispensable for biofilm control. All of these procedures were meant to miniminize the halitosis and accumulation of calculus. Besides that, we encouraged the child to drink water often, in order to miniminize the dry mouth sensation, because she was already used to drink water and other liquids normally and she had medical consent for this.

Follow-up for every three months showed that the patient and her parents perceived a improvement on her oral health and they did not claim anymore about

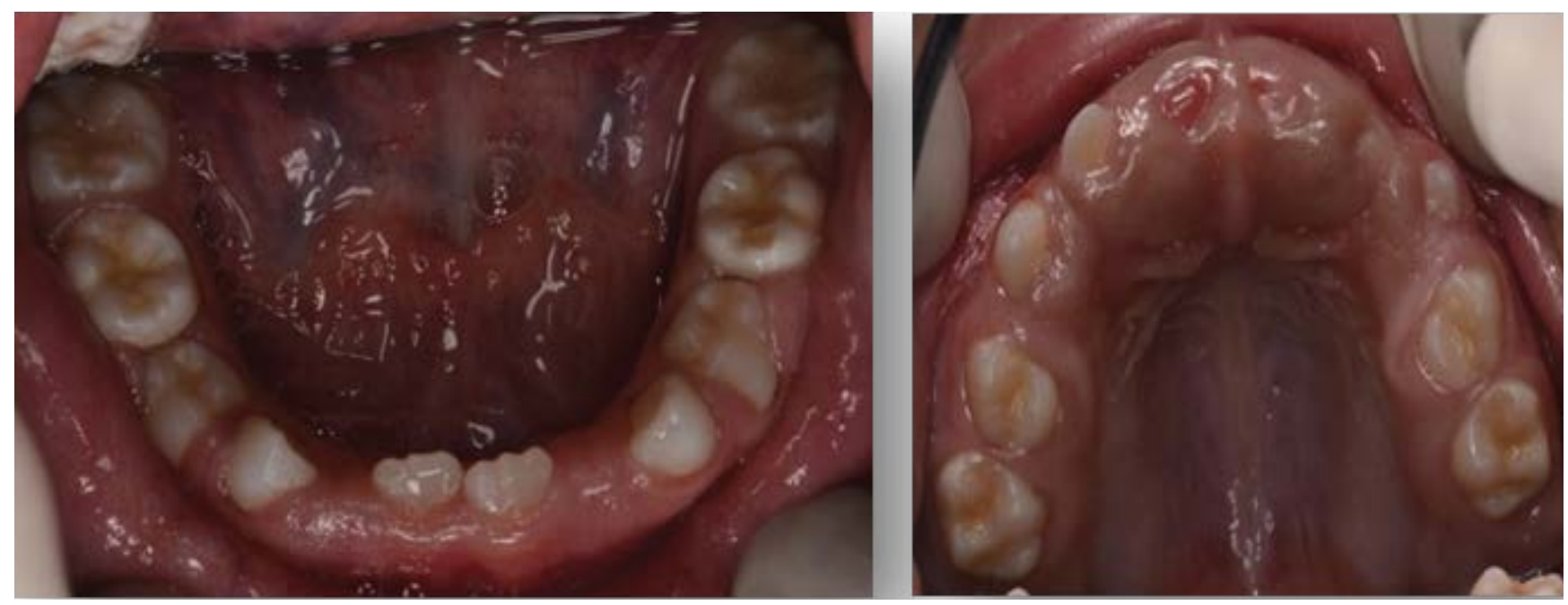

Figures 3: $(A$ and $B)$ Clinical status showing recent exfoliation in the upper arch and infra-occlusion of the first primary molars in the lower.

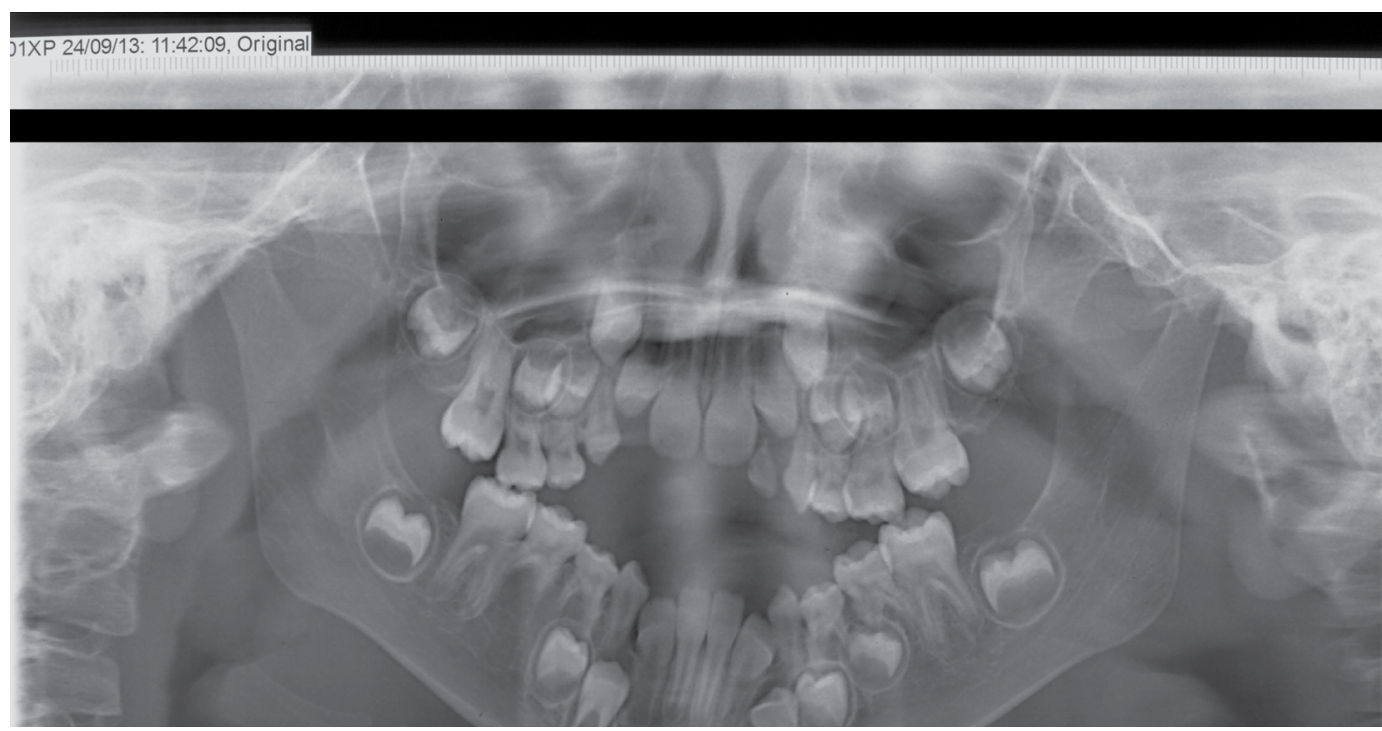

Figure 4: Panoramic Radiography showing wide anterior open bite and absence of dental element 45. 
the bad breath. She was referred then to the surgery department of the same institution for evaluation and orthognathic planning in the future, when she is older than 18-years-old.

\section{Discussion}

The merosine - deficient form of CMD is traditionally known as a homogeneous disease, with severe hypotonic muscles, multiple contractures, delayed developmental milestones and normal intelligence accompanied by variable degrees of central hypomyelination on cerebral imagining [8]. But there are some cases in the literature, where mental retardation in CMDMC patients is described, most of them related to consanguineous parents. Talim, et al. and Hui, et al. $[9,10]$ reported two cases of both twins, brother and sister diagnosed with CMDMC with normal intelligence and both were able to play computer games and to speak full sentences. In this report, the patient (twin sister of a child with no systemic disease) has normal intelligence, is also able to play, and expresses herself very well.

Little is known about the oral status of MDCMD patients. In regard to the oral stomatognathic system, the medical literature about MDCMD patients related some reports about the absence of sucking reflex during the breastfeeding period, which it may be then an indicatory to the diagnosis of MDCMD and it must be investigated $[4,5,7]$. Our patient also presented the absence of sucking reflex at 10-months-old and needed to be underwent the surgery of gastric fed tube placement, since the suction is necessary for natural breast feeding.

Besides, frequent feeding problems in merosin deficient congenital muscular dystrophy is related by many authors for example, difficulty chewing the food, abnormal swallowing of the bolus with or without aspiration, and gastroesophageal reflux $[4,7,11]$. Our patient also had recurrent infections and probably also due to aspiration the difficulties of swallowing and little sucking reflex during the breastfeeding period. Philpot, et al. [11] conducted a study with 14 children, which showed the importance of considering the nutritional status in any child with a primary muscle disorder. They affirmed that the children with MDCMD have difficulties at all stages of feeding that progress with age and they showed that the appropriate intervention could improve weight gain and reduce chest infections. They also related that the mouth architecture was abnormal in 13 of all 14 children. Our patient also had an abnormal mouth architecture, which does not allow her to close the mouth correctly.

Besides that, her exacerbated anterior open bite is a predisposing factor to traumatic dental injuries. Moreover, traumatic dental injury prevalence in children with disability is higher than that of non-disabled children [12].

Feeding difficulties can be distressing for the indi- vidual and their family. Prolonged mealtimes and concern about poor weight gain with continual emphasis on diet lead to psychological problems, especially as the child gets older [11]. Our patient started consuming soft food, liquids and candies after she had cognitive development, after the age of 4-years-old, but the parents are still very apprehensive about the swallowing reflex.

About halitosis, there is scientific evidence that etiology of halitosis is usually from the oral cavity. The volatile sulfur compounds produced by some of the oral bacteria are responsible for its development. Only $10 \%$ of the causes are extraoral, mostly inflammation of airways or gastrointestinal disorders. Beyond the dental treatments, the enhancement of the oral hygiene, the continuous motivation and monitoring are also very important, such as the use of tongue cleansing, special anti-malodour rinses and sugar-free chewing gums $[13,14]$. With our patient, besides those recommendations, it was also highlighted the importance of oral hygiene, such as teeth brushing, dental floss use and tongue scrapping and we observed improvement on halitosis complaint.

Jones and Waites [1] reported a case of a 14-yearold girl with MDCMD presenting apertognathia, who underwent an orthognathic surgery and partial glossectomy to improve her quality of life, due to her main complaints of an enlarged tongue with protrusion from the oral cavity resulting in tongue dryness and sunburns, an impaired eating ability caused by the patient's malocclusion and macroglossia and a severely concave profile. In our case, the child was not able to swallow perfectly and also presented the apertognathia, but probably only due to her condition of MDMDC, which may be diagnosed through the oral status, because it is peculiar to the condition. Our patient is still very young to undergo such a surgery, so the best approach at this age is to solve the chief complaints at this time until the surgery can be performed.

\section{Conclusion}

Patients with MDCMD present several oral manifestations, however, the dentist is able to approach and improve a better quality of life related to oral health to them, since is essential for children. A pediatric dentist should monitor the children since birth, in order to minimize the chief complaints and highlight the importance of oral hygiene.

\section{References}

1. Lewis C Jones, Peter D Waite (2012) Orthognathic Surgery and Partial Glossectomy in a Patient with Merosin- Deficient Congenital Muscular Dystrophy. J Oral Maxillofac Surg 70: e141-e146.

2. Mostacciuolo ML, Miorin M, Martinello F, Angelini C, Perini P, et al. (1996) Genetic epidemiology of congenital muscular dystrophy in a sample from north-east Italy. Hum Genet 97: 277. 
3. Allamand V, Guicheney P (2002) Merosin-deficient congenital muscular dystrophy, autosomal recessive (MDC1A MIM\#156225, LAMA2 gene coding for alpha2 chain of laminin). Eur J Hum Genet 10: 91.

4. Mercuri E, Sewry C, Brown SC, Muntoni F (2002) Congenital muscular dystrophies. Semin Pediatr Neurol 9: 120-131.

5. Ralte AM, Sharma MC, Gulati S, Das M, Sarkar C (2003) Merosin negative congenital muscular dystrophy: a short report. Neurology India 51: 417-419.

6. Ueki K, Nakagawa K, Yamamoto E (2007) Bite force and maxillofacial morphology in patients with Duchenne-type muscular dystrophy. J Oral Maxillofac Surg 65: 34.

7. Elena Buteica, Eugenia Rosulescu, F Burada, B Stanoiu, Mihaela Zavaleanu (2008) Merosin-Deficient Congenital Muscular Dystrophy Type 1a. Rom J Morphol Embryol 49: 229-233.

8. Mendel Jr (2001) Congenital muscular dystrophy: searching for a definition after 98 years. Neurology 56: 993-994.
9. Talim B, Kale G, Topaloglu H, Akcoren Z, Caglar M, et al. (2000) Clinical and histopathological study of merosin-deficient and merosin-positive congenital muscular dystrophy. Pediatr Dev Pathol 3: 168-176.

10. CM Hui, Kwong L, Lam SY, Loo KT (2004) Merosin-deficient congenital muscular dystrophy in two siblings. Hong Kong Med J 10: 423-426.

11. J Philpot, A Bagnall, C King, V Dubowitz, F Muntoni (1999) Feeding problems in merosin deficient congenital muscular dystrophy. Arch Dis Child 80: 542-547.

12. Murthy AK, Chandrakala B, Pramila M, Ranganath S (2013) Dental trauma in children with disabilities in India: a comparative study. Eur Arch Paediatr Dent 14: 221-225.

13. Zalewska A, Zatonski M, Jablonka-Strom A, Paradowska A, Kawala B, et al. (2012) Halitosis--a common medical and social problem. A review on pathology, diagnosis and treatment. Acta Gastroenterol Belg 75: 300-309.

14. Scully C (2014) Halitosis. BMJ Clin Evid 1305. 